\title{
RELEVO DEL MANDATO DEL GOBERNADOR DEL BANCO CENTRAL NACIONAL Y PAPEL DEL TRIBUNAL DE JUSTICIA: LA INDEPENDENCIA JUSTIFICA EL CONTROL. COMENTARIO A LA SENTENCIA DEL TRIBUNAL DE JUSTICIA (GRAN SALA) DE 26 DE FEBRERO DE 2019, RIMŠÉVIČS Y BCE/LETONIA
}

\author{
PAZ ANDRÉS SÁENZ DE SANTA MARÍA ${ }^{1}$ \\ Universidad de Oviedo \\ pandres@uniovi.es
}

Cómo citar/Citation

Andrés Sáenz de Santa María, P. (2019).

Relevo del mandato del gobernador del banco central nacional y papel del Tribunal de Justicia: la independencia justifica el control.

Comentario a la sentencia del Tribunal de Justicia (Gran Sala) de 26 de febrero de 2019, Rimšēvičs y BCE/Letonia.

Revista de Derecho Comunitario Europeo, 63, 629-649.

https://doi.org/10.18042/cepc/rdce.63.08

\section{Resumen}

Ante una situación inédita como es la prohibición provisional del ejercicio de funciones dictada por una autoridad administrativa nacional respecto del gobernador del banco central de Letonia, el Tribunal de Justicia aborda por primera vez

1 Catedrática de Derecho Internacional Público de la Universidad de Oviedo. 
la naturaleza y alcance del recurso regulado en el art. 14.2, párr. segundo, de los Estatutos del SEBC y del BCE. Con el fin de garantizar la independencia y el buen funcionamiento del SEBC, el TJ afirma su competencia mediante una interpretación amplia del concepto de relevo del mandato y se pronuncia sobre la naturaleza del procedimiento, considerando que se trata de un recurso específico de anulación dentro del sistema de recursos establecido por los Tratados. Ante la ausencia de indicios suficientes del fundamento de las acusaciones formuladas contra el gobernador, el Tribunal considera que Letonia no ha probado la existencia de falta grave y anula el acto nacional, lo que supone una decisión sin precedentes que se justifica por la construcción jurídica original que constituye el SEBC.

\title{
Palabras clave
}

Art. 14.2, párr. segundo, de los Estatutos del SEBC y del BCE; relevo de mandato; acto nacional; competencia del TJ; recurso de anulación; independencia del gobernador del banco central nacional; falta grave.

\section{RELIEF FROM OFFICE OF THE GOVERNOR OF THE NATIONAL CENTRAL BANK AND ROLE OF THE COURT OF JUSTICE: INDEPENDENCE JUSTIFIES THE CONTROL. COMMENTARY OF THE JUDGMENT OF THE COURT (GRAND CHAMBER) OF 26 FEBRUARY 2019, RIMŠÉVIČS AND ECB/LATVIA}

\begin{abstract}
Faced with an unprecedented situation, such as the provisional prohibition of the exercise of functions by a national administrative authority with respect to the governor of the central bank of Latvia, the Court of Justice addresses for the first time the nature and scope of the remedy regulated in art. 14.2, second subparagraph, of the Statute of the ESCB and the ECB. In order to ensure the independence and proper functioning of the ESCB, the CJ affirms its jurisdiction by a broad interpretation of the concept of relief from office and decides on the nature of the procedure, considering that it is a specific action of annulment within the system of legal remedies laid down by the Treaties. In the absence of any evidence capable of establishing the existence of sufficient indications as regards whether the accusations made against the governor, the court considers that Latvia has not proven the existence of serious misconduct and annuls the national act, which is an unprecedented decision that is justified by the novel legal construction which constitutes the ESCB.
\end{abstract}

\section{Keywords}

Article 14.2, second subparagraph, of the Statute of the European System of Central Banks and of the European Central Bank; relief from office; national act; 
jurisdiction of the Court; action for annulment; independence of the governor of the national central bank; serious misconduct.

\section{RELÈVEMENT DE FONCTIONS DU GOUVERNEUR DE LA BANQUE CENTRALE NATIONALE ET RÔLE DE LA COUR DE JUSTICE: L'INDÉPENDANCE JUSTIFIE LE CONTRÔLE. COMMENTAIRE SUR L'ARRÊT DE LA COUR (GRANDE CHAMBRE) DU 26 FÉVRIER 2019, RIMŠÉVIČS ET BCE/ LETTONIE}

\section{Résumé}

Face à une situation sans précédent, telle que l'interdiction provisoire de l'exercice des fonctions exercées par une autorité administrative nationale à l'égard du gouverneur de la Banque centrale de Lettonie, la Cour de Justice examine pour la première fois la nature et l'étendue du recours prévu à l'art. 14.2, deuxième alinéa, des Statuts du SEBC et de la BCE. Afin de garantir l'indépendance et le bon fonctionnement du SEBC, la CJ affirme sa compétence par une interprétation large de la notion de relèvement de fonctions et décide de la nature de la procédure, estimant qu'il s'agit d'un recours spécifique en annulation dans le système des voies de recours prévu par les Traités. En l'absence d'élément de preuve de nature à établir l'existence d'indices suffisants quant au bien-fondé des accusations portées contre le gouverneur, la cour estime que la Lettonie n'a pas prouvé l'existence d'une faute grave et annule l'acte national, ce qui constitue une décision sans précédent et justifiée par la construction juridique originale qui constitue le SEBC.

\section{Mots clés}

Article 14.2, second alinéa, des Statuts du Système européen de banques centrales et de la Banque centrale européenne; relèvement de fonctions; acte nationale; compétence de la Cour; recours en annulation; indépendance du gouverneur du banque central national; faute grave. 


\section{SUMARIO}

I. INTRODUCCIÓN. II. LA COMPETENCIA DEL TRIBUNAL DE JUSTICIA: PECULIARIDADES: 1. La importancia de la calificación de la medida a efectos de determinar la competencia del Tribunal de Justicia. 2. La irrelevancia de la dimensión penal interna. 3. El aspecto no contemplado: la determinación del grado de jurisdicción. III. LA NATURALEZA DEL RECURSO: ¿̇ECLARAR O ANULAR?: 1. Un recurso específico de anulación vinculado a las características del SEBC. 2. El alcance de la calificación como recurso de anulación: ¿̇el SEBC o incluso más allá? IV. EL CONTROL DE LA LEGALIDAD DEL ACTO ESTATAL: LA NECESIDAD DE PROBAR LA EXISTENCIA DE INDICIOS SUFICIENTES DE FALTA GRAVE. V. UNA PRIMERA VEZ QUE DEJA HUELLA. Bibliografía.

\section{INTRODUCCIÓN}

¿Bajo qué condiciones pueden los Estados miembros de la Unión Europea relevar de sus funciones a los gobernadores de sus bancos centrales? Aunque la abogada general Kokott ${ }^{2}$ resumió así la cuestión que se planteaba en los asuntos planteados por el gobernador del banco de Letonia y el Banco Central Europeo (BCE) en relación con la resolución por la que la Oficina de Prevención y Lucha contra la Corrupción de ese Estado prohibió provisionalmente al Sr. Rimšēvičs ejercer sus funciones de gobernador y abandonar el país por estar sometido a un procedimiento penal por presunta corrupción, la sentencia del Tribunal de Justicia (Gran Sala) de 26 de febrero de $2019^{3}$ se adentra en aspectos de envergadura que alcanzan dimensión constitucional y desbordan aquella síntesis.

2 Conclusiones de la abogada general Kokott, Rimšēvičs y Banco Central Europeo/ Letonia, C-202/18 y C-238/18, EU:C:2018:1030, punto 1. Todas las fuentes electrónicas utilizadas han sido consultadas por última vez el 1 de mayo de 2019.

3 C-202/18 y C-238/18, EU:C:2019:139. Se trata de asuntos acumulados y tramitados con arreglo al procedimiento acelerado; esta decisión la tomó el presidente del TJ a solicitud del BCE en el asunto C-238/18 (Auto de 12 de junio de 2018, BCE/Letonia, EU:2018:488) y de oficio en el asunto 202/18 (Auto de 12 de junio de 2018, Rimšēvičs/Letonia, EU:2018:489). 
De esta forma, la decisión — precedida por un auto sobre medidas provisionales ${ }^{4}$ - se une a otras que viene adoptando en los últimos tiempos para resolver asuntos «difíciles legalmente y sensibles políticamente» (Rosas, 2919: $7)^{5}$. No cabe duda de que este lo era, tanto por ser la primera vez en la que se invocaba el art. 14.2, párr. segundo, del Protocolo sobre los Estatutos del Sistema Europeo de Bancos Centrales y del Banco Central Europeo (en adelante, Estatutos) como por el particular contexto fáctico del caso, en el que el gobernador letón es acusado de aceptar presuntamente sobornos en beneficio de un banco privado ${ }^{6}$, la medida adoptada contra él es provisional y el procedimiento no ha finalizado cuando se dicta la sentencia. La conjunción de estos elementos coloca al Tribunal de Justicia (TJ) ante problemas de competencia, de determinación de la naturaleza del recurso y de control de legalidad en un ámbito, el de los bancos centrales nacionales, en el que las competencias de cada Estado miembro han de compaginarse con su integración en el Sistema Europeo de Bancos Centrales (SEBC).

\section{LA COMPETENCIA DEL TRIBUNAL DE JUSTICIA: PECULIARIDADES}

La habitual cuestión previa de la competencia del tribunal presenta en esta ocasión aspectos peculiares, de un lado por la singularidad de la medida nacional adoptada, de otro por la dimensión penal del caso; también, por la

4 Auto de 20 de julio de 2018, BCE/Letonia, C-238/18 R, EU:C:2018:581. En él, el vicepresidente del TJ ordenó a Letonia suspender las medidas temporales adoptadas con relación al Sr. Rimšẽvičs en la medida en que le impedían designar un suplente para sustituirlo como miembro del Consejo de Gobierno del BCE. Sobre este auto puede verse Kovar y Lasserre (2018: 79-81).

5 Como señala el juez Rosas, «the European Court has increasingly been asked to perform the functions of a constitutional court that has to decide burning issues of considerable constitutional and political importance, and this takes place in the framework of a constitutional order which is dynamic and far from settled» (Rosas, 2019: 2).

6 Se trataba de Trasta Komercbanka, presuntamente implicado en blanqueo de dinero al que se le retiró la licencia y fue disuelto; las decisiones adoptadas en este caso por el BCE han sido objeto de varios recursos ante el TJUE interpuestos por el Consejo de Administración y los accionistas (véanse los casos Fursin y otros /BCE, T-247/16, EU:T:2017:263; Trasta Komercbanka y otros/BCE, T-698/16; BCE/Trasta Komercbanka y otros, C-663/17 P; Comisión/ Trasta Komercbanka y otros y BCE, C-665/17 P; Trasta Komercbanka y otros/BCE, C-669/17 P). 
imprecisa expresión utilizada en el art. 14.2, párr. segundo, de los Estatutos respecto al grado de jurisdicción competente dentro de la institución judicial.

\section{LA IMPORTANCIA DE LA CALIFICACIÓN DE LA MEDIDA A EFECTOS DE DETERMINAR LA COMPETENCIA DEL TRIBUNAL DE JUSTICIA}

La citada disposición consagra el derecho del gobernador afectado y del Consejo de Gobierno del BCE a recurrir cuando aquel sea «relevado de su mandato" por dejar de cumplir los requisitos exigidos para el cumplimiento de sus funciones o haya incurrido en falta grave. Pero la resolución de la Oficina de Prevención y Lucha contra la Corrupción de Letonia era una medida provisional adoptada para garantizar la instrucción efectiva de la investigación, lo que dio pie a Letonia a argumentar que la única resolución que podría ser recurrida sería la que en su caso pusiera fin al vínculo jurídico e institucional entre el gobernador y el banco central nacional, mientras que la medida controvertida era temporal y podría ser revocada. Según el Estado, los conceptos de «relevo de mandato» utilizado en el art. 14.2, párr. segundo, y de "cese» usado en otras disposiciones ${ }^{7}$ son equivalentes, además de que según su legislación la institución competente para relevar del mandato al gobernador es el Parlamento, el cual no ha adoptado ninguna decisión.

$\mathrm{El} \mathrm{TJ}$ reconoce que los términos del art. 14.2, párr. segundo, "parecen referirse $[\ldots]$ a la ruptura definitiva del vínculo entre el banco central nacional y su gobernador ${ }^{8}$, pero una interpretación sistemática y teleológica vinculada al principio de la independencia del SEBC le lleva a entender que la prohibición temporal a un gobernador de un banco central nacional de ejercer sus funciones puede constituir un medio de presión sobre él que afecte a su independencia.

Esa interpretación y ese principio habían sido invocados tanto por el BCE como por la abogada general para rechazar una visión restrictiva y justificar el encaje de la resolución disputada en la disposición de referencia? Como no podía ser de otra manera, la demanda del BCE exponía en detalle el significado y la importancia de la independencia y defendía una versión

\footnotetext{
Arts. 246, 247 y 286 TFUE, 11.4 de los Estatutos del SEBC y del BCE y 26.4 del Reglamento (UE) n. ${ }^{\circ}$ 1024/2013, del Consejo, de 15 de octubre de 2013, que encomienda al Banco Central Europeo tareas específicas respecto de políticas relacionadas con la supervisión prudencial de las entidades de crédito.

8 Apdo. 44.

9 De manera menos razonada, el Sr. Rimšēvičs también sostuvo que la prohibición de ejercer sus funciones de gobernador por una duración no especificada constituye un relevo del mandato (véase el apdo. 2 de la sentencia).
} 
amplia del concepto de relevo del mandato para incluir en él las medidas que obstaculicen el ejercicio de las funciones que le confiere el derecho de la Unión, cualquiera que sea su duración ${ }^{10}$. Por su parte, Kokott se apoyó también en el efecto útil del art. 14.2, párr. segundo, y en el principio de independencia para considerar que una medida nacional pueda ser objeto de control por el TJ si tiene como efecto impedir al gobernador el ejercicio de sus funciones con independencia de su clasificación formal en el derecho nacional, propugnando estar a su sustancia y a sus efectos concretos ${ }^{11}$. Ambos alertaban del peligro del uso torticero por un Estado de medidas temporales que en la práctica tuvieran un efecto equivalente, si se entendiera que no eran susceptibles de control jurisdiccional ${ }^{12}$.

La sentencia comparte este enfoque, recordando que los autores de los Tratados «tuvieron la voluntad de garantizar que el BCE y el SEBC pudieran

10 Como sintetiza el TJ: «El BCE arguye que, habida cuenta de la finalidad de esta disposición, una prohibición de ejercer cualquier función correspondiente al puesto de gobernador de un banco central nacional, aunque no extingue formalmente el vínculo jurídico e institucional de este último con dicho banco, debe considerarse equivalente a un relevo del mandato, en el sentido del artículo 14.2, párrafo segundo, de los Estatutos del SEBC y del BCE. Si se aplicara la interpretación contraria, los Estados miembros, al adoptar medidas de esa naturaleza, podrían eludir la garantía de independencia prevista por este texto. La independencia del gobernador se ve igualmente afectada por la prohibición temporal al gobernador afectado de ejercer sus funciones, a fortiori cuando, como en el presente asunto, se ignora cuándo finalizará la prohibición, hecho que puede producirse después de la expiración del mandato del gobernador» (apdo. 42). La argumentación del BCE sobre este tema fue uno de los elementos tomados en consideración por el vicepresidente del TJ para apreciar fumus boni juris en el auto de medidas provisionales (Auto de 20 de julio de 2018, BCE/ Letonia, C-238/18 R, EU:C:2018:581, apdos. 37-38).

11 Véanse los puntos 74-77 de las Conclusiones.

12 En palabras de la abogada general: «However, the temporary nature of a measure or the fact that it does not have the effect of definitively severing the legal and institutional link between the governor and the national central bank concerned does not preclude that measure from being classified as relieving the governor from office within the meaning of Article 14.2 of the Statute of the ESCB and of the ECB, if it actually has the consequence of preventing the governor from performing his duties. Were that not so, the Member States would be able to circumvent the prohibition laid down in that provision by adopting measures not formally classified as 'relieving from office' but having an equivalent effect in practice. Furthermore, as the ECB correctly states, what appears to be a temporary measure may turn out to be definitive if the effects last until the end of the term of office of the governor concerned» (punto 79). 
cumplir con independencia las funciones que les asignan ${ }^{13}$. En su argumentación, el TJ relaciona directamente el art. 14.2, párr. segundo, de los Estatutos con la garantía de la independencia de los gobernadores ${ }^{14}$, vincula el control jurisdiccional con su protección y asume que «si una medida que prohíbe a un gobernador desempeñar sus funciones pudiera eludir cualquier control del Tribunal de Justicia con arreglo al artículo 14.2, párrafo segundo, de los Estatutos del SEBC y del BCE, debido a que es temporal, sería fácil para un Estado miembro, adoptando medidas temporales consecutivas, eludir este control, por lo que [...] dicha disposición podría quedar privada de efecto útil $»^{15}$. Por todo ello, el Tribunal se considera competente para conocer de recursos contra medidas como la impugnada.

Del razonamiento contenido en la decisión, conviene destacar dos aspectos. En primer lugar, la medida provisional susceptible de control jurisdiccional, aunque no constituya un relevo formal del mandato, tiene que tener las características reunidas por la resolución adoptada por la Oficina de Prevención y Lucha contra la Corrupción de Letonia, esto es, que con independencia de su forma y del órgano que la adopte, produzca en la práctica un efecto equivalente al relevo del mandato, en particular cuando la medida no tenga fecha de finalización precisa, se pueda aplicar durante una parte significativa del mandato o pueda acabar teniendo efectos definitivos habida cuenta del tiempo restante para la finalización del mandato en el caso concreto ${ }^{16}$.

En segundo lugar, el bien objeto de protección es la independencia del gobernador en el ejercicio de sus funciones vinculadas al SEBC, entre las que el TJ destaca la participación en el Consejo de Gobierno del BCE. En este sentido, no es casualidad que afirme que la finalidad esencial de las disposiciones aplicables "consiste, en sustancia, en mantener al SEBC al abrigo de todas las presiones políticas a fin de permitirle perseguir eficazmente los objetivos asignados a sus misiones, mediante el ejercicio independiente de las facultades específicas de que dispone al efecto en virtud del Derecho

13 Apdo. 46.

14 El Tribunal utiliza la expresión «independencia funcional» (apdos. 48 y 70), aunque en realidad se está refiriendo a lo que habitualmente se califica como independencia personal, esto es, las garantías relacionadas con el nombramiento de los miembros de los órganos del BCE y el SEBC, su relevo y desempeño de las atribuciones durante el mandato, mientras que la independencia funcional alude al poder de decisión autónomo para utilizar los instrumentos y adoptar las medidas necesarias para el cumplimiento de sus fines. Sobre los distintos elementos de la independencia véase Smits (1997: 155-158).

15 Apdo. 53.

16 Véanse los apdos. 51 y 54. 
primario» ${ }^{17}$ y que «si pudiera decidirse relevar de su mandato a los gobernadores de los bancos centrales nacionales sin tener que justificarlo, su independencia se vería seriamente comprometida y, en consecuencia, la del propio Consejo de Gobierno del BCE» ${ }^{18}$.

A lo anterior cabe añadir que desde esta perspectiva, esta sentencia se une a otras en las que con anterioridad se ha pronunciado sobre la independencia del BCE: los asuntos $O L A F^{19}$, Gauweiler ${ }^{20}$ y Weiss $^{21}$. Si entonces se trataba de la independencia institucional y funcional, ahora se añade la dimensión personal ${ }^{22}$. Se confirma de este modo la valoración según la cual

Europe's law on the independence of the central bank is the strictest set of rules possible. Drawing inspiration from the template of the Bundesbank [...] and written with the intention of fending off intervention into monetary policy by politicians from Member States with less of a tradition of central bank independence, the Treaty and Statute downright prohibit influencing those entrusted with setting and implementing Europe's monetary policy (Smits, 2007:1624).

\section{LA IRRELEVANCIA DE LA DIMENSIÓN PENAL INTERNA}

En su defensa, Letonia intentó sacar partido a la dimensión penal del caso, argumentando la vinculación de la resolución controvertida con el procedimiento incoado, la exclusión de la competencia del TJUE establecida

7 Apdo. 47.

18 Apdo. 51. La preocupación por asegurar el buen funcionamiento del Consejo de Gobierno del BCE está muy presente en el auto de medidas provisionales (véanse los apdos. 71-76); también lo está en el auto por el que se decide la aplicación del procedimiento acelerado (véase el apdo. 12).

19 Sentencia del Tribunal de Justicia de 1 de julio de 2003, Comisión/BCE, C-11/00, EU:C:2003:395, apdos. 130-145. Véanse también las Conclusiones del abogado general Jacobs, Comisión/BCE, C-11/00, EU:C:2002:556, puntos 147-155.

20 Sentencia del Tribunal de Justicia de 16 de junio de 2015, Gauweiler, C-62/14, EU:C:2015:400, apdo. 40. Véanse también las Conclusiones del abogado general Cruz, Gauweiler, C-62/14, EU:C:2015:7, puntos 108-109. Como advierte Baroncelli (2016: 83-84), aunque la decisión solo se refiere brevemente a la independencia, "a closer reading of the case reveals that central bank independence is the 'hidden concept' which underpins the reasoning of the Court of Justice, so much that it is possible to justify the conclusions reached by the Court of Justice in the case on the basis of the notion of central bank independence».

21 Apdos. 49 y 60.

22 Véase la descripción que de este aspecto de la independencia hace el abogado general Jacobs en el asunto OLAF, EU:C:2002:556, punto 153. 
en el art. 276 TFUE e incluso que el reconocimiento de esta competencia supondría conceder una inmunidad penal al gobernador, al tiempo que incidiría en la sustanciación del procedimiento penal. Ninguno de estos motivos es tomado en consideración por el Tribunal, al igual que tampoco lo habían sido por la abogada general ${ }^{23}$. La sentencia recuerda que el derecho de la Unión impone límites a la competencia de los Estados miembros en materia penal, de forma que estas deben ejercerse observando no solo las libertades fundamentales garantizadas por aquel derecho, sino también el ordenamiento de la Unión en su conjunto, en particular el derecho primario, por lo que «las normas de procedimiento penal nacional no pueden ser un obstáculo a la competencia que el artículo 14.2, párrafo segundo, de los Estatutos del SEBC y del BCE atribuye al Tribunal de Justicia en todos los casos en que sea aplicable esta disposición ${ }^{24}$, descarta que la medida litigiosa entre dentro del ámbito de materias contempladas por el art. 276 TFUE $^{25}$ y rechaza cualquier interferencia en el ámbito penal estatal.

En este punto, la sentencia denota un especial cuidado en deslindar las competencias del TJ respecto de las del Estado y fijar los límites de las primeras. Así, señala que la "eventual concurrencia» solo se produciría en el supuesto excepcional en el que un procedimiento penal conduzca a adoptar respecto de un gobernador de un banco central nacional una medida provisional que pueda asimilarse a un relevo del mandato y que incluso en tal hipótesis el recurso del art. 14.2, párr. segundo, de los Estatutos no obstaculizaría la instrucción de la investigación pena ${ }^{26}$. El interés del Tribunal por dejar claro este tema le lleva a añadir, tras proclamar su competencia, la siguiente precisión:

La competencia del Tribunal de Justicia en virtud de dicha disposición está limitada a los recursos contra una prohibición temporal o definitiva de ejercer las funciones de gobernador de un banco central nacional. En consecuencia, el Tribunal de Justicia solo es competente para conocer de la resolución impugnada, mediante la cual el KNAB adoptó varias medidas vinculantes, en lo que atañe a la prohibición, de carácter temporal, impuesta al Sr. Rimšěvičs de ejercer sus funciones de gobernador del Banco de Letonia ${ }^{27}$.

23 Véanse los puntos 81-86 de las Conclusiones.

24 Apdo. 57.

25 Así se habían pronunciado también el vicepresidente en el Auto de medidas provisionales (apdos. 28-29) y la abogada general en sus Conclusiones (puntos 81-82).

26 Apdo. 61.

27 Apdo. 63. 


\section{EL ASPECTO NO CONTEMPLADO: LA DETERMINACIÓN DEL GRADO DE JURISDICCIÓN}

A diferencia de la mayoría de las disposiciones del TFUE relativas a los recursos, que se refieren al "Tribunal de Justicia de la Unión Europea» salvo los arts. 269 y $273^{28}$, el art. 14.2, párr. segundo, de los Estatutos alude al «Tribunal de Justicia», lo que abre la cuestión del grado de jurisdicción competente. En su demanda, el BCE interpretó la mención específica como una reserva de competencia que excluye al Tribunal General. A su vez, la abogada general llegó a la misma conclusión, considerándolo como un procedimiento específico frente a los procedimientos judiciales generales del BCE ante las jurisdicciones de la Unión, en los que se aplican las reglas habituales de reparto de competencias, y lo justificó así:

The allocation of jurisdiction to hear and determine the specific action provided for in Article 14.2 of the Statute of the ESCB and of the ECB to the Court of Justice is justified in the light of the constitutional importance of the independence of the governors of the central banks and the political sensitivity of questions relating to their being relieved from office. Last, the need to resolve a dispute concerning the decision to relieve a governor from office quickly in order to ensure the proper functioning of the ESCB and the ECB argues against the establishment of two levels of jurisdiction for the action concerning such a decision ${ }^{29}$.

Siendo pertinente el planteamiento de la cuestión y atinada la justificación proporcionada por la abogada general, en su sentencia el TJ no se detiene en ella, se entiende que por considerar obvia su competencia, en una interpretación ad pedem litterae.

\section{LA NATURALEZA DEL RECURSO: ¿̇DECLARAR O ANULAR?}

Hay que reconocer que hasta el presente el art. 14.2, párr. segundo, de los Estatutos había merecido poca atención por parte de la doctrina, limitándose la mayoría a enunciar su contenido. Con todo, algunos especialistas habían destacado su singularidad ${ }^{30}$. Así, Zilioli y Selmayr (2001:

28 También se refieren al TJUE los arts. 35 y 36 de los Estatutos; en cambio, el art. 11.4 cita específicamente al TJ.

29 Punto 71.

30 Como recuerda Sarmiento Ramírez-Escudero (2019): «This provision is, in the words of a dear colleague, an 'extravagant rule', unparalleled in the Treaties». 
78) apuntaron: "This is the first time ever that a "national" authority has been allowed to challenge its own Member State in a supranational court», mientras que ya con anterioridad Smits (1997: 165) había advertido:

A direct appeal to the European Court against a national decision... is a novelty which crept into Community law through the backdoor route of EMU provisions. Normally, only Community decisions can be appealed against before the Court of Justice, national measures either being 'tried' in the procedure of Articles 169 and 170 or coming before the Court through a reference by a national court for a preliminary ruling (Article 177). The appeal to the European Court is only on one of the four grounds enumerated in Article 173 EC Treaty, the provision on legal review of acts of the institutions of the Community.

Sin embargo, en las escasas referencias valorativas, es difícil encontrar pronunciamientos sobre la naturaleza del recurso consagrado en esa disposición. La excepción viene proporcionada por Louis (2009: 175-176), quien avanza: «On s'est interrogé sur la nature des pouvoirs de la Cour, qui doit être saisie dans un délai de deux mois. Peut-ell annuler l'acte national ou doit-elle se borner à constater le manquement? Compte tenu de la spécificité des relations entre $\mathrm{BCN}$ et $\mathrm{BCE}$ à l'intérieur du Système, il ne semble pas que l'on doive dans ce cas exclure une annulation».

Ciertamente, la calificación presenta dificultades porque, como han señalado Kovar y Lasserre (2019: 92):

La question de la nature de cette voie de recours est d'autant plus délicate que celle-ci présente des particularités qui la distinguent tant du recours en manquement que du recours en annulation. D’une part, la voie de recours de l'article 14.2 est ouverte au gouverneur concerné et au conseil des gouverneurs de la BCE, alors que le recours en manquement prévu à l'article 258 TFUE peut être exercé par la Commission et les autres États membres. D'autre part, l'article 14.2 permet de contester devant la Cour un acte d'un État membre, alors que le recours en annulation de l'article 263 TFUE ne peut être dirigé qu'à l'encontre des actes adoptés par les institutions, les organes ou les organismes de l'Union.

La sentencia objeto de este comentario, en lo que seguramente es su aportación de mayor trascendencia, zanja el dilema considerándolo como un recurso de anulación. 


\section{UN RECURSO ESPECÍFICO DE ANULACIÓN VINCULADO A LAS CARACTERÍSTICAS DEL SEBC}

Aunque los dos recursos que han dado lugar a esta decisión pedían al TJ pronunciamientos declarativos ${ }^{31}$, la abogada general abordó en sus Conclusiones el tema de la naturaleza del recurso, apuntando las implicaciones teóricas y prácticas de la respuesta; en lo que atañe a estas últimas, señaló:

The practical implications of the answer to the question as to the nature of the present actions are not insignificant: if the Court were to annul the decision of the KNAB imposing the restrictive measures at issue on Mr Rimšēvičs, he would be able to resume office immediately after the Court delivered its judgment. If, on the other hand, the Court were merely to find that the measures in question were incompatible with the Statute of the ESCB and of the ECB, it would be for the Republic of Latvia to take the necessary measures to ensure that the judgment of the Court was implemented within its internal legal order ${ }^{32}$.

Tras un detallado análisis del art. 14.2, párr. segundo, de los Estatutos, teniendo en cuenta sus términos, la economía general y el contexto de la regulación así como sus facilidades objetivos y génesis, Kokott llegaba a la conclusión de que no se trata de un recurso de anulación sino de "constatación", semejante al recurso por incumplimiento; en particular, la consideración de la existencia de dos esferas en el sistema de recursos ante el TJUE, una para las instituciones, órganos y organismos de la Unión y otra para los Estados, sus órganos e instituciones, le hacía pensar que:

The annulment of an act taken by a national authority would constitute not only an unusual but also an extremely serious interference in the sphere of competence and the procedural autonomy of the Member States. In the light of the constitutional importance of the principles of subsidiarity and of the conferral of powers enshrined in Articles 4 and 5 TEU, the possibility of such interference would have to be expressly provided for in the Treaties ${ }^{33}$.

31 Véanse los apdos. 19-20 y 64, en los que aparece un dato interesante a los efectos que nos ocupan pues el Tribunal refiere que: «Preguntado en la vista sobre la naturaleza del recurso previsto en el artículo 14.2, párrafo segundo, de los Estatutos, el BCE precisó que solicitaba que el Tribunal de Justicia pronuncie "una sentencia declarativa, como es el caso en los procedimientos de infracción”»; véanse asimismo los anuncios de los recursos (DO C 161, de 7 de mayo de 2018, pp. 43-44).

32 Punto 39.

33 Punto 60. La argumentación de la abogada general se encuentra en los puntos 36-62 de las Conclusiones. 
Por el contrario, en un giro que ha sorprendido a muchos, el Tribunal - como ya se ha apuntado - lo califica en cambio como recurso de anulación, conclusión a la que llega también mediante una interpretación literal, sistemática y teleológica de la disposición. En esta operación, de un lado, encuentra en su tenor semejanzas con el citado recurso en lo que se refiere a la legitimación activa de un particular, el plazo de dos meses para la interposición y los motivos invocables; de otro, lo cataloga como un recurso específico que se añade al sistema de recursos establecido por los Tratados, que se justifica por las particularidades del SEBC; además, toma en cuenta la finalidad de garantizar la independencia y el buen funcionamiento del Consejo de Gobierno del $\mathrm{BCE}$, del que el gobernador del banco central nacional forma parte. Todo ello le lleva a afirmar que

únicamente el recurso de anulación, completado, en su caso, por las medidas cautelares que puede ordenar el Tribunal de Justicia con arreglo a los artículos 278 TFUE y 279 TFUE, permite responder a las preocupaciones que llevaron a la creación de este recurso. En particular, no se daría respuesta de manera satisfactoria a las intenciones de los autores de los Estatutos del SEBC y del BCE si la sentencia dictada en virtud del artículo 14.2, párrafo segundo, de dichos Estatutos tuviera carácter declarativo y si sus efectos dependieran de su ejecución por parte de las autoridades nacionales ${ }^{34}$.

En coherencia con la decisión adoptada sobre la naturaleza del recurso, el TJ estima que al haber sido presentados sobre la base del art. 14.2, párr. segundo, de los Estatutos, «los recursos interpuestos por el Sr. Rimšēvičs y el BCE tienen por objeto la anulación de la resolución impugnada».

El cambio cualitativo que entraña la posición del Tribunal habrá hecho las delicias de los demandantes pero explica el sobresalto experimentado por algunos autores ${ }^{35}$. No obstante, el Tribunal proporciona claves convincentes que justifican el resultado al que llega. En efecto, pone de relieve que la disposición en cuestión establece una excepción al reparto general de competencias entre el juez nacional y el juez de la Unión, la cual

34 Apdo. 74. La posibilidad que la Comisión siempre tiene abierta de interponer un recurso por incumplimiento conforta también la posición adoptada por el TJ, quien advierte de que «no puede admitirse que, al establecer la vía de recurso prevista en el artículo 14.2, párrafo segundo, de los Estatutos del SEBC y del BCE, los autores de dicha disposición pretendieran simplemente crear un procedimiento paralelo al ya previsto en el artículo 258 TFUE» (apdo. 75).

35 En palabras de Costa (2019): «With all due respect, the ruling comes as a big surprise». 
se explica por el contexto institucional concreto del SEBC, del que forma parte. En efecto, el SEBC constituye en Derecho de la Unión una construcción juridica original en la que participan y cooperan estrechamente las instituciones nacionales, esto es, los bancos centrales nacionales, y una institución de la Unión, a saber, el BCE, y en el que prima una articulación diferente y una distinción menos pronunciada entre el ordenamiento jurídico de la Unión y los ordenamientos jurídicos internos"

[...] el artículo 14.2 de los Estatutos del SEBC y del BCE materializa las consecuencias de ese sistema, muy integrado, deseado por los autores de los Tratados para el SEBC y, en particular, del desdoblamiento funcional del gobernador de un banco central nacional, ciertamente autoridad nacional que actúa en el marco del SEBC y que, cuando es gobernador de un banco central nacional de un Estado miembro cuya moneda es el euro, forma parte del principal órgano de dirección del BCE. Debido a este estatuto híbrido y, como se ha señalado en el apartado 48 de la presente sentencia, de manera excepcional, a fin de garantizar la independencia funcional de los gobernadores de los bancos centrales nacionales en el SEBC, una decisión adoptada por una autoridad nacional por la que se releva a uno de ellos de su mandato puede ser sometida al Tribunal de Justicia ${ }^{36}$.

En este escenario singular, el recurso es una de las garantías de que el gobernador desempeñará sus funciones con independencia, y representa "una pieza esencial del equilibrio institucional que requiere la estrecha cooperación de los bancos centrales nacionales y el BCE en el SEBC ${ }^{37}$.

Con esta argumentación, el TJ sintetiza los rasgos esenciales del sistema integrado de política monetaria consagrado en los Tratados y los proyecta sobre el sistema de recursos, extrayendo las consecuencias sobre la naturaleza de un recurso que hasta el momento había pasado desapercibido entre el articulado de los Estatutos del SEBC y del BCE y que ahora aflora manifestándose como una excepción a la distribución de competencias entre la jurisdicción europea y las nacionales.

En realidad, la doctrina especializada ya había apuntado las ideas que acoge la sentencia. Así, al destacar el carácter peculiar del art. 14.2, párr. segundo, Zilioli y Selmayr apuntaron (2001: 78): «All this shows that inside the ESCB, the national central banks no longer act as national authorities, but as agents of the $E S C B »$, añadiendo:

The integration of the national central banks into the ESCB has led to a «denationalisation» of most of the national central banks' functions and to a «communaut-

36 Apdos. 69-70. Cursiva añadida.

37 Apdo. 72. 
arisation» of the national central banks - a development which represents the most far-reaching example of a "dédoublement fonctionnel» ever witnessed in Community law and which shows again the close link between the ECB and the national central banks inside the organisational structure of the ESCB (2001: 79-80).

En definitiva, lo que hace el Tribunal es poner negro sobre blanco las características del sistema y mostrar un rasgo práctico de la dimensión procesal de esa «construcción jurídica original» que es el SEBC.

\section{EL ALCANCE DE LA CALIFICACIÓN COMO RECURSO DE ANULACIÓN: ¿̇EL SEBC O INCLUSO MÁS ALLÁ?}

Casi no hace falta destacar las consecuencias del «descubrimiento» de este recurso específico que emerge en lo que hasta la actualidad era un pacífico sistema con dos esferas bien determinadas en las que la anulación solo opera en relación con las instituciones de la Unión ${ }^{38}$. Ahora sabemos que en el ámbito del SEBC el TJ puede anular las decisiones estatales relacionadas con el relevo del mandato del gobernador del banco central nacional.

Por ello, se ha destacado que «there is a direct road of redress to Luxembourg instead of an indirect route» (Smits, 2019) y también se ha resaltado la relevancia que ha tenido el principio de independencia en la decisión: «Independence has been a key factor in deciding Rimševičss, a factor which came with the cost to strike down a nacional decision by the CJEU in order to safeguard it» (Costa, 2019). Entre nosotros, Sarmiento (2019) describe de forma impresionista el resultado:

The annulment or repeal of national law is not left to the national authorities, but quite the contrary: it is left to the Court to purge the rule itself, irrespective of the author or position of the authority rendering the rule. If the rule would have been a constitutional provision, the Court would annul. If the rule would have been a judgment of a national court, the Court would annul. The fact that the annulled act was an individual decision of an administrative body does not deprive the judgment of its seismic impact.

En su opinión, la decisión de anular el acto estatal relativo al gobernador letón «hides a revolution with the potential of changing EU law forever» $y$ entiende que puede no tratarse de una decisión aislada:

38 Véase la descripción que la abogada general hace de estas dos esferas en sus Conclusiones, puntos 54-55. 
Thus, one could argue that this result is confined to the European System of Central Banks and no more. But think twice. "Novel legal constructs" are constantly in the making in the EU. Just take a glimpse at the recently created European Prosecutor's Office, in which another "novel legal construct" has been put into place to safeguard the financial interests of the EU. If in the future the Court strikes down a national criminal judgment on the grounds that the European Prosecutor's Office is a "novel legal construct" within which "a different structure and a less marked distinction between the EU legal order and national legal orders prevail”, the assertion's premiss would be correct. The same applies to the Single Supervisory Mechanism, whereby the ECB implements national law, and national authorities enforce EU law and ECB instructions. Novel legal constructs are not the exclusive domain of monetary policy, and the challenges of European integration are blending EU and national legal orders in unprecedented ways that blur the distinction between both systems (Sarmiento, 2019).

Otros, en cambio, consideran que el alcance no irá más allá del SEBC: «The Court emphasises the specific, unique and excepcional nature of the remedy, so the judgement is not opening the floodgates to similar leapfrogging of national proceedings by going directly to the CJEU» (Smits, 2019); "the Court's ruling is exceptional and closely related to the EU's monetary regime» (Costa, 2019); «il faut donc se garder de toute interprétation hâtive et voir dans cette jurisprudence le moment constitutionnel de la Cour de justice de l'Union européenne puisqu'elle s'autoriserait à un contrôle des mesures nationales. Il s'agit bien d'un contrôle de légalité des mesures nationales mais il est limité au cadre spécifique du SEBC. Le principe reste celui selon lequel le juge de l'Union européenne ne peut apprécier la validité des normes étatiques» (Pignarre, 2019: 9).

En este sentido, en el estado actual de la integración europea no se vislumbra la posibilidad de que se materialice un proyecto de características similares a las constatadas por el TJ en relación con la unión monetaria. A lo largo de la motivación, la sentencia hace hincapié en la excepcionalidad del recurso y sus consecuencias, llegando a aludir al «escaso número de personas que pueden interponerlo, el objeto único de las resoluciones contra las cuales está previsto y las circunstancias excepcionales en los que cabe interponerlo» ${ }^{39}$. No obstante, cosas veredes...

39 Apdo. 71. Para Kovar y Lasserre (2019: 92): «Cette dérogation à la répartition générale des compétences entre le juge national et le juge de l'Union s'explique par le contexte particulier du SEBC, formé par les banques centrales nationales et la BCE, et le dédoublement fonctionnel du gouverneur d'une banque centrale d'un pays de la 


\section{EL CONTROL DE LA LEGALIDAD DEL ACTO ESTATAL: LA NECESIDAD DE PROBAR LA EXISTENCIA DE INDICIOS SUFICIENTES DE FALTA GRAVE}

Aclarado el sentido del recurso, procedía determinar en cuál de los dos supuestos previstos en el art. 14.2, párr. segundo, de los Estatutos encajaban los hechos que dieron lugar a la medida temporal impuesta al Sr. Rimšēvičs, operación en la que tanto el BCE como la abogada general y el TJ coinciden en estimar que se trataría de una presunta falta grave. Ello conduce a la cuestión de la prueba, que para el BCE requeriría el examen por un tribunal independiente ${ }^{40}$ aunque admitía no obstante que en circunstancias excepcionales el relevo del mandato podría producirse antes de que se dictara una sentencia condenatoria ${ }^{41}$ y para la abogada general exigiría un juicio sobre el fondo pronunciado por un tribunal independiente o la presentación de elementos de prueba suficientes para que el TJ alcance por sí mismo una convicción firme ${ }^{42}$.

El Tribunal se preocupa por deslindar su ámbito de competencia en relación con la existencia de la falta grave en el sentido del art. 14.2 de los Estatutos. Al respecto, aclara que no le corresponde «sustituir a los tribunales nacionales competentes para pronunciarse sobre la responsabilidad penal del gobernador investigado ni tampoco interferir en la investigación penal preliminar», y admite que para impedir la obstrucción de la investigación puede ser necesario suspenderle temporalmente en sus funciones; lo que sí le corresponde es "comprobar que únicamente se adopte una prohibición provisional al gobernador afectado de ejercer sus funciones si existen indicios suficientes de que ha incurrido en una falta grave que pueda justificar tal medida ${ }^{43}$. Sentado lo anterior, la escasez y debilidad de las pruebas aportadas por Letonia llevan al TJ a declarar que este Estado no ha acreditado la existencia de indicios suficientes de falta grave y a estimar el motivo basado en el carácter injustificado de la medida adoptada contra el gobernador, procediendo a anular la resolución de la Oficina de Prevención y Lucha contra la Corrupción de Letonia en la medida en que prohíbe a aquel ejercer sus funciones.

zone euro, autorité nationale siégeant au sein du principal organe de décision d'une institution de l'Union» .

40 Para esta institución, esta exigencia permite garantizar el respeto del derecho a un juicio justo y la presunción de inocencia (véase el apdo. 84).

41 Véase el apdo. 85.

42 Véanse los puntos 107-108.

43 Apdos. 91-92. Según Pignarre (2019: 6), la referencia a los «indicios suficientes» evoca un control limitado al error manifiesto de apreciación. 
El reconocimiento de la posibilidad de la suspensión temporal de funciones de un gobernador de un banco central nacional cierra el círculo abierto por el Tribunal al inclinarse por un concepto amplio del relevo del mandato con el que avala su competencia: para proteger la instrucción penal, el Estado podría suspender temporalmente al gobernador en sus funciones ${ }^{44}$, pero este acto es susceptible de control por el TJ para comprobar si existen indicios suficientes que lo justifiquen.

Por lo demás, destaca la parquedad de esta parte final de la sentencia, omitiendo cualquier referencia a los perfiles del concepto de falta grave y a los requisitos de las pruebas y no pronunciándose, por ejemplo, sobre si sería exigible la intervención de un tribunal independiente, en la línea apuntada por el $\mathrm{BCE}^{45}$ y la abogada general; al respecto cabe intuir que el TJ admitiría que la medida provisional se adopte sobre la base de la actuación de una autoridad gubernamental siempre que los indicios probatorios sean suficientes ${ }^{46}$. Es verdad que la práctica ausencia de pruebas por parte de Letonia le permi-

44 Ya el auto del vicepresidente sobre medidas provisionales no acogió la solicitud del BCE de suspender la medidas temporal de suspensión de funciones del gobernador letón para permitirle cumplir, en su calidad de miembro del Consejo de Gobierno, las tareas no vinculadas con el objeto de la investigación penal, por entender que semejante medida podría atentar gravemente contra la efectividad de la encuesta penal (véanse los apdos. 78-79).

45 El auto sobre medidas provisionales parece acoger el criterio del BCE, cuando afirma: «D'autre part, n'apparaît pas non plus manifestement infondé l'argument de la BCE selon lequel la constatation d'une faute grave au sens de l'article 14.2, second alinéa, du protocole sur le SEBC et la BCE suppose, en principe, que le gouverneur concerné ait fait l'objet d'une condamnation prononcée au fond par un tribunal indépendant et qu'une décision adoptée par un organe d'enquête ne soit donc pas suffisante à cette fin. En effet, une telle interprétation de cette disposition pourrait, à première vue, sembler mieux à même de garantir que la décision relevant ledit gouverneur de ses fonctions repose sur des motifs étayés et constatés par un tribunal indépendant au cours d'une procédure offrant toutes les garanties attachées au droit à une protection juridictionnelle effective, en vue d'assurer l'indépendance des gouverneurs des banques centrales nationales» (apdo. 39).

46 La abogada general había alertado sobre la fiabilidad de los hechos alegados por una autoridad administrativa: «Unlike in the case of facts established by a judgment on the merits delivered by an independent tribunal, the Court cannot accept as established facts which are alleged to have taken place only by administrative authorities. Such authorities do not enjoy the same guarantees of institutional and functional independence as the courts and do not take their decisions following an adversarial procedure together with the guarantees of an effective remedy. Accepting facts alleged by an administrative authority as established without ascertaining that they are correct would 
tían ahorrarse el sentar doctrina sobre estos aspectos, pero también lo es que si lo hubiera hecho, habría redondeado una decisión tan relevante como en todo caso es la que comentamos.

\section{UNA PRIMERA VEZ QUE DEJA HUELLA}

Era la primera vez que el TJ era llamado a pronunciarse sobre la base del art. 14.2, párr. segundo, de los Estatutos del SEBC y del BCE y el Tribunal, una vez más, no ha desaprovechado la oportunidad para crear otro «momento constitucional» (Sarmiento, 2019). Lo ha hecho en un contexto fáctico espinoso, porque cuando se dicta la sentencia el resultado final de las investigaciones todavía no se conoce y también porque las presuntas responsabilidades en las que podría haber incurrido el gobernador del banco central letón no pueden ser más incompatibles con el honrado desempeño de sus funciones. Esto explica asimismo la cautela con la que se desempeñó el BCE, atrapado entre la obligación de defender los principios estructurales de su estatuto y el temor de un resultado adverso en el procedimiento penal nacional.

El asunto ha sacado a la luz un precepto peculiar desde el mismo momento de su redacción. En sus esfuerzos por reconstruir su génesis, la abogada general apenas pudo rememorar que los Estatutos fueron redactados por los gobernadores de los bancos centrales de los Estados miembros con algún añadido en el último momento y que, en lo que atañe a esta disposición, no hay ninguna traza de debates ${ }^{47}$. Sin ataduras derivadas de la génesis del texto y con un tenor literal susceptible de ser interpretado tanto en términos de anulación como de declaración, el TJ se ha inclinado por la solución más protectora del SEBC y al mismo tiempo más coherente con este particular ámbito en el que la independencia se erige en principio estructural del sistema, las instituciones nacionales desdoblan sus funciones y las fronteras entre el derecho de la Unión y los ordenamientos estatales se difuminan. La consecuencia colateral es la histórica anulación de un acto estatal.

El controvertido gobernador letón ha contribuido sin pretenderlo a la historia del control jurisdiccional en la Unión Europea, dando pie a una sentencia que dejará huella. Con todo, por el bien de la credibilidad del Eurosistema, confiemos en que ningún otro gobernador se sienta tentado a proporcionar nuevas oportunidades de esta índole.

therefore amount to depriving the parties concerned of their right of effective judicial review» (punto 134).

47 Véanse los puntos 45-50. 


\section{Bibliografía}

Baroncelli, S. (2016). The Gauweiler Judgement in View of the Case Law of the European Court of Justice on European Central Bank Independence: Between Substance and Form. Maastricht Journal of European and Comparative Law, 23 (1), 79-98. Disponible en: https://doi.org/10.1177/1023263X1602300105.

Costa, M. (2019). Accountability and independence of the Governors of National Banks: Any role for the Court of Justice of the European Union? Eulawanalysis [blog], 25-3-2019. Disponible en https://bit.ly/2HXucZL.

Kovar, J. P. y Lasserre Capdeville, J. (2018). Indépendance de la Banque centrale européenne et des gouverneurs des banques centrales nationales. Revue Banque, 825, 79-81.

Kovar, J. P. y Lasserre Capdeville, J. (2019). Indépendance des gouverneurs des banques centrales et de la BCE. Revue Banque, 831, 90-92.

Louis, J. V. (2009). L’Union européenne et sa monnaie. Bruxelles: Éditions Université de Bruxelles.

Pignarre, P. E. (2019). Anatomie d'une première: Le contrôle de légalité d'une mesure nationale par la CJUE. Blogdroiteuropeen Working Paper [blog], 9-5-2019. Disponible en https://bit.ly/2ERo17x.

Rosas, A. (2019). The European Court of Justice: Do all roads lead to Luxemboug? CEPS Policy Insights, 2019/3. Disponible en: https://bit.ly/2NIFG4P.

Sarmiento, D. (2019). Crossing the Baltic Rubicon. Despite our Differences [blog], 4-3-2019. Disponible en: https://bit.ly/2QOf97D.

Smits, R. (1997). The Central European Bank. Institutional Aspects. The Hague; London; Boston: Kluwer Law International.

— (2007). The European Central Bank's Independence and Its Relations with Economic Policy Makers. Fordham International Law Journal, 31 (6), 16121636.

- (2019). ECJ annuls a national measure against an independent central banker. European Law Blog [blog], 5.3.2019. Disponible en https://bit.ly/2WeTzdD.

Zilioli, C. y Selmayr, M. (2001). The Law of the European Central Bank. Oxford; Portland Oregon: Hart Publishing. 